\title{
Glycosyltransferase-Mediated Exchange of Rare Microbial Sugars with Natural Products
}

\author{
Ramesh P. Pandey ${ }^{1,2 *}$ and Jae K. Sohng ${ }^{1,2 *}$ \\ ${ }^{1}$ Department of BT-Convergent Pharmaceutical Engineering, Sun Moon University, Asan-si, South Korea, ${ }^{2}$ Department of \\ Life Science and Biochemical Engineering, Sun Moon University, Asan-si, South Korea
}

Keywords: glycosyltransferase, glycodiversification, glycosylation, natural products, rare microbial sugar

OPEN ACCESS

Edited by:

Javier González-Sabín,

EntreChem, Spain

Reviewed by:

Shawn Chen,

Revive Genomics Inc., USA

Carlos Olano,

Universidad de Oviedo, Spain

*Correspondence:

Ramesh P. Pandey

pandey@sunmoon.ac.kr

Jae K. Sohng

sohng@sunmoon.ac.kr

Specialty section:

This article was submitted to

Microbiotechnology, Ecotoxicology

and Bioremediation,

a section of the journal

Frontiers in Microbiology

Received: 20 September 2016 Accepted: 03 November 2016

Published: 16 November 2016

Citation:

Pandey RP and Sohng JK (2016)

Glycosyltransferase-Mediated

Exchange of Rare Microbial Sugars

with Natural Products.

Front. Microbiol. 7:1849.

doi: 10.3389/fmicb.2016.01849
A large number of plant and microbial architectures have been identified and investigated for potential applications in therapeutics, cosmetics, and nutrition. Nonetheless, continuous identification, and design of novel lead molecules are prerequisites to tackling emerging diseases since most existing drugs are losing utility because of resistance by microorganisms. Advances in biotechnological tools in systems and synthetic biology, chemical biology and metabolic engineering, genome sequencing, and synthesis, protein engineering and mutagenesis have enabled alteration of the biological routes of natural product (NP) biosynthesis in heterologous robust hosts to produce a wide array of compounds (Pandey et al., 2016), thus adding diversity in the NPs. Post-modifications of NPs by tailoring enzymes is one of the promising approaches for engineering and manipulating NPs under human control with selective power. Glycosyltransferases (GTs) have been attracting tremendous attention because of their potential to diversify NPs by conjugating diverse types of sugar appendages (Williams et al., 2007, 2011; Pandey et al., 2014), and altering the physico-chemical and biological properties, such as adsorption, distribution, metabolism, and excretion of molecules (Weymouth-Wilson, 1997). For example, when mycosamine sugar was replaced by perosamine in amphotericin $\mathrm{B}$, antifungal and hemolytic activities were improved in new derivative which has minimal inhibitory activity concentration (MIC) of $1.9 \mu \mathrm{g} / \mathrm{ml}$ compared to $2.1 \mu \mathrm{g} / \mathrm{ml}$ of amphotericin B against Saccharomyces cerevisiae (Hutchinson et al., 2010). Similarly, when D-desosamine of YC-17 was replaced with four sugars D-quinovose, L-rhamnose, L-olivose, and D-boivinose, the L-rhamnose sugar conjugated derivative exhibited better antibacterial activity than the parent YC-17 against erythromycin-susceptible and resistant Enterococcus faecium and Staphylococcus aureus (Shinde et al., 2013). GTs have also been exploited to reverse the glycosylation reactions in NPs (Zhang et al., 2006). This property of GTs expanded the possibility of synthesizing diverse nucleotide diphosphate (NDP)-sugars and exchanging them among different classes of NPs by single vessel trans-glycosylation (Zhang et al., 2014).

The application of GTs in the industrial biosynthesis of NP glycosides (De Bruyn et al., 2015), detoxification of pollutants, pesticides, and xenobiotics (Stupp et al., 2013), and homeostasis of plant hormones to control crop engineering (Tiwari et al., 2016) has profound impact on human daily life. Thus, plant and microbial GTs with broad substrate promiscuity have been identified and characterized to glycodiversify NPs to further widen their scope for the generation of future molecules for human use (Elshahawi et al., 2015; Tiwari et al., 2016). Such powerful enzymes can be engaged for the exchange of diverse sugar moieties using microbial cells engineered to produce secondary metabolites using metabolic engineering tools.

Most of the plant glycosylated secondary metabolites contain unmodified simple sugars, such as D-glucose, D-galactose, D-glucuronic acid, L-rhamnose, D-xylose, and D-arabinose and many more. Thus, plant GTs are usually studied for their catalytic potential to harness the aforementioned simple sugar moieties for a diverse class of metabolites, such as polyphenols, terpenoids, benzoates, salicyclic acids, and polyketides. In contrast to plant glycosides, microbial originated glycosylated 
molecules contain highly modified deoxysugars with different functional moieties, such as amino-, nitro-, keto-, and sulfogroups in their structures. These modified sugar moieties are found to play a crucial role in executing biological functions to those microbial metabolites, such as doxorubicin, staurosporine, vancomycin, calicheamicin, amphotericin B, tylosin, and erythromycin (Kren and Rezanka, 2008).

GT-mediated exchange of sugar moieties of microbial origin with secondary metabolites in metabolically engineered hosts is a fascinating viable approach for the generation of novel biologically potent compounds (Chen, 2011). This approach can be achieved in two different ways as shown in Figure 1. The first approach is to supplement aglycone along with a chemically modified synthesized sugar in an engineered microbial cell harboring anomeric kinase (AK), nucleotidyl transferase (NT), and a promiscuous GT. All three enzymes should be capable of harnessing diverse sets of sugars, sugar phosphates, and NDP-sugars, respectively. AK phosphorylates exogenously supplemented sugar to sugar-1-phosphate, which is subsequently utilized by NT to produce NDP-sugar at an expense of NTP. Thus, the generated NDP-sugar is utilized by GT as a donor substrate and the sugar moiety is transferred to exogenously supplemented aglycone/acceptor molecules. The newly generated glycosides are usually excreted outside of the cell into the culture medium and are readily extracted and purified. In contrast to the first approach, the second in vivo glycosylation platform does not require the supplementation of chemically synthesized sugars. Nevertheless, rare NDP-sugars are produced in the cell cytosol by heterologous expression of diverse sets of sugar cassettes. These rare sugars are finally transferred to exogenously supplemented aglycones by substrate promiscuous GT. The production of diverse NDP-sugars in the cell cytosol by both approaches glycodiversify the exogenously added molecule.

Such green approaches for the in vivo glycodiversification of aglycones by diverse sugar appendages are superior to existing in vitro strategies. The necessity of multiple enzyme production and purification for enzymatic synthesis of NDP-sugars, low yield of purified target NDP-sugars, cofactor addition or regeneration during the process of reaction usually increases the process cost for in vitro glycorandomization (Fu et al., 2004). On the other hand, the chemical approach for NP glycosides synthesis is tedious, hazardous, time consuming, and requires protection and de-protection of the functional groups. Since there is a high chance of by-products formation, the purification of molecules is difficult, which ultimately lowers the production yield of the target molecule. Thus, the microbial approach of conjugating diverse sugars to aglycones is the simplest, cheapest, and easiest approach that can be readily scaled up to industrial level by simply fermenting engineered microbes and supplementing aglycones and sugars as required (Song et al., 2013). However, in vivo glycorandomization approach needs further development because some of the aglycone substrates fed to the microbial culture system require specific transporters for entry and exit of the substrates and products through the membrane, respectively. This problem of substrate transportation could be addressed by overexpression of specific transporter proteins to the host organisms. Additionally, endogenous NDP-sugars or free monosaccharides (first approach Figure 1) compete with target unnatural sugar that has to be transferred to aglycone during in vivo glycosylation reaction. Rewiring of endogenous sugar pathways in host cell by metabolic engineering could overcome this problem of substrates competition in the cell cytosol.

Simple and high-throughput screening methods are prerequisite to identify sugar-exchanged products and to access the activities of engineered GTs in glycosylation reactions. However, very few such analytical methods are developed to detect glycosylated products at relatively low concentration. Some of the high-throughput methods used in glycosylation reactions are (i) use of fluorescent compounds as acceptor substrates whose fluorescence signal decreases upon sugar conjugation (Gantt et al., 2008), (ii) fluorescence-activated cell sorting (FACS) analysis used to identify cells in which fluorescent labeled sugars conjugated molecules are entrapped (Yang et al., 2010), (iii) enzyme-linked immunosorbent assay (ELISA) based detection of glycosylated products using carbohydrate-binding proteins (Hancock et al., 2009), and (iv) pH based color change of indicator molecule in the course of glycosylation reaction (Park et al., 2009). Besides these methods, the most abundantly used analytical approach for glycosylation reactions is highperformance liquid chromatography (HPLC). HPLC could be coupled with high resolution mass spectrometry (HR/MS) for direct identification of the NP-glycosides. HPLC based analyses of glycosylation reactions are not applicable for high-throughput screening of large number of GTs and their mutants. Hence, the development of a more efficient high-throughput screening approach for GT-mediated glycosylation reactions is obligatory.

Recently, advances have been made in the construction of robust microbial cells for the synthesis of several biomolecules in E. coli, S. cerevisiae, and Streptomyces. These biosynthesis approaches include de novo pathway engineering, central carbon flux redirection for co-factor or precursor synthesis, the selection of alternative enzymes, protein engineering/mutagenesis, precursor directed mutasynthesis, modular metabolic engineering, system and synthetic biology tools, such as codon optimization, vector, promoter, ribosome binding site engineering, and the engineering and manipulation of other non-protein-coding sequences (Pandey et al., 2016). For microbial production of NP glycosides, though different enzymes sources, particularly GTs, are used, no system/synthetic biology tools are applied for co-factor regeneration in microbial cell cytosol. Flux analysis for co-factor regeneration and optimum cell growth maintenance is crucial for efficient biosynthesis of glycosides. Several pathway genes for rare NDP-sugars, particularly thymidine diphosphate (TDP)-sugars, can be heterologously expressed for the modification of TDP-4-keto6-deoxy-D-glucose (TKDG), which is the major intermediate of TDP-deoxysugars (Thibodeaux et al., 2008). Fine tuning of all possible heterologously expressed genes is essential for the balanced production of final NDP-sugars. In most cases for the biosynthesis of non-natural glycosides, the major limiting factor is the production of NDP-sugar in the cell cytosol of E. coli. This hurdle can be addressed by regulating intermediate consuming pathway genes in the chromosome of host organisms. Several 


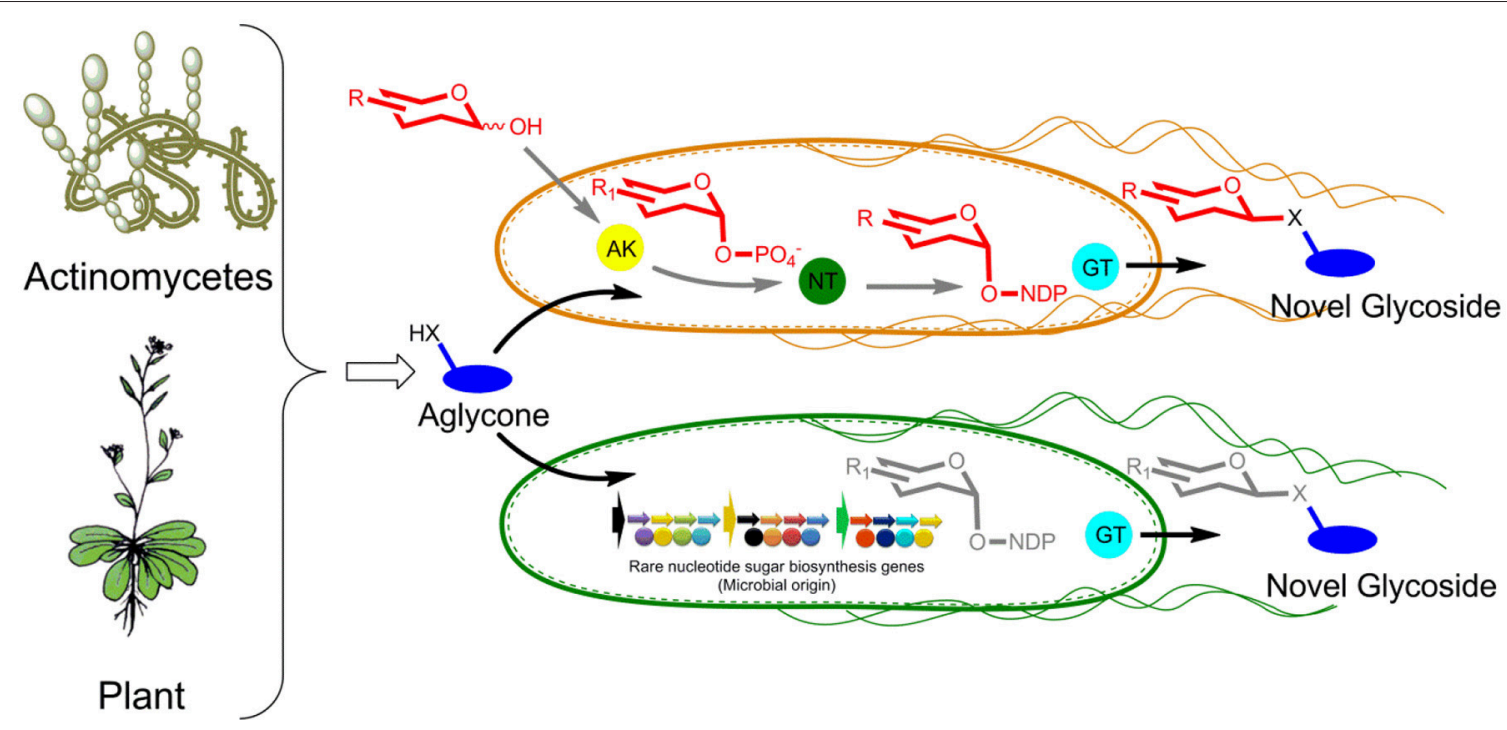

FIGURE 1 | GT-mediated microbial glycone and aglycone exchange platforms for NPs diversification by glycosylation $(\mathrm{X}=\mathrm{O}, \mathrm{S}$, or $\mathrm{NH})$. In first strain, reducing sugars and aglycones are fed while in second strain only aglycones are fed. AK, anomeric kinase; NT, nucleotidyl transferase; GT, glycosyltransferase.

studies have been reported by our group (Malla et al., 2013; Pandey et al., 2015) and other researchers to block alternate biosynthesis pathway genes in E. coli by constructing background E. coli mutants (Kim et al., 2015). However, the complete knockdown of essential genes for cell wall biosynthesis hampers cell growth and necessitates additional precursors to supplement the intermediates of blocked pathways. Thus, to rule out these problems, we should apply recently developed gene regulation tools, such as clustered regularly interspaced short palindromic repeats interference (CRISPERi) (Larson et al., 2013) and sRNA mediated gene regulation systems (Yoo et al., 2013) to control the flow of NDP-sugar biosynthesis intermediates to undesired products without hampering cell health. Moreover, application of these tools can also maintain high cell density during fermentation. Beside these options, the adequate expression of heterologous genes in the engineered host is another important aspect for rare NDP-sugar biosynthesis. To balance the fine tuning of all gene expression, modular and multivariate NDPsugar biosynthesis pathways should be constructed to address the accumulation of an intermediate NDP-sugar in the biosynthesis pathway. Besides the biosynthesis of rare NDP-sugars in the microbial cell cytosol, we also need to focus on GT engineering for the generation of promiscuous GT that can accept diverse sets of NDP-sugars and acceptors for forward glycosylation reactions. Only a few GTs have been studied in detail for broad applications to NP glycodiversification. This is limited because of the lack of sufficient GTs with crystal structures. Thus, further study should be focused on the screening of highly promiscuous GTs and their design/engineering based on crystal structure and computer based modeling approaches, saturation mutagenesis after identification of "hot spots" for directed evolution. Such engineered mutant GTs would have high potential to generate a wide array of glycodiversified NPs. Eventually upon the biosynthesis of a library of novel NP glycosides, those molecules could be accessed for possible applications in therapeutics, cosmetics, and nutraceutics.

The in vivo fermentation approach for producing NP glycosides is inadequate for producing a significant amount of glycosides by simple fermentation even though the process can be easily subjected to industrial scale up. To produce future NP glycosides by fermentation, a robust system should be developed that is capable of producing diverse NDP-sugars in the cell cytosol while engineering promiscuous GTs for sugar transfer to acceptor molecules. High level production of target non-natural NP glycosides can be achieved by simple microbial fermentation upon proper implementation of recently developed system/synthetic biology tools for the engineering of microbial host cells. Cumulatively, this approach of GT-mediated exchange of microbial non-natural glycones with exogenous aglycones offers huge combinatorial potential for the biosynthesis of novel future molecules for human use.

\section{AUTHOR CONTRIBUTIONS}

$\mathrm{RP}$ and JS wrote the manuscript.

\section{ACKNOWLEDGMENTS}

This work was supported by the National Research Foundation of Korea (NRF) grant funded by the Korea government (MEST) (NRF-2014R1A2A2A01002875). 


\section{REFERENCES}

Chen, X. (2011). Fermenting next generation glycosylated therapeutics. ACS Chem. Biol. 6, 14-17. doi: 10.1021/cb100375y

De Bruyn, F., Maertens, J., Beauprez, J., Soetaert, W., and De Mey, M. (2015). Biotechnological advances in UDP-sugar based glycosylation of small molecules. Biotechnol. Adv. 33, 288-302. doi: 10.1016/j.biotechadv.2015. 02.005

Elshahawi, S. I., Shaaban, K. A., Kharel, M. K., and Thorson, J. S. (2015). A comprehensive review of glycosylated bacterial natural products. Chem. Soc. Rev. 44, 7591-7697. doi: 10.1039/C4CS00426D

Fu, X., Langenhan, J. M., and Thorson, J. S. (2004). Combinatorial chemoenzymatic strategies for in vitro glycorandomization: efforts toward antibiotic optimization. Discov. Med. 4, 111-114.

Gantt, R. W., Goff, R. D., Williams, G. J., and Thorson, J. S. (2008). Probing the aglycon promiscuity of an engineered glycosyltransferase. Angew. Chem. Int. Ed. Engl. 47, 8889-8892. doi: 10.1002/anie.200803508

Hancock, S. M., Rich, J. R., Caines, M. E., Strynadka, N. C., and Withers, S. G. (2009). Designer enzymes for glycosphingolipid synthesis by directed evolution. Nat. Chem. Biol. 5, 508-514. doi: 10.1038/ nchembio.191

Hutchinson, E., Murphy, B., Dunne, T., Breen, C., Rawlings, B., and Caffrey, P. (2010). Redesign of polyene macrolide glycosylation: engineered biosynthesis of 19-(O)-perosaminyl-amphoteronolide B. Chem. Biol. 17, 174-182. doi: 10.1016/j.chembiol.2010.01.007

Kim, B. G., Yang, S. M., Kim, S. Y., Cha, M. N., and Ahn, J. H. (2015). Biosynthesis and production of glycosylated flavonoids in Escherichia coli: current state and perspectives. Appl. Microbiol. Biotechnol. 99, 2979-2988. doi: 10.1007/s00253015-6504-6

Kren, V., and Rezanka, T. (2008). Sweet antibiotics-the role of glycosidic residues inantibiotic and antitumor activity and their randomization. FEMS Microbiol. Rev. 32, 858-889. doi: 10.1111/j.1574-6976.2008.00124.x

Larson, M. H., Gilbert, L. A., Wang, X., Lim, W. A., Weissman, J. S., and Qi, L. S. (2013). CRISPR interference (CRISPRi) for sequence-specific control of gene expression. Nat. Protoc. 8, 2180-2196. doi: 10.1038/nprot.2013.132

Malla, S., Pandey, R. P., Kim, B. G., and Sohng, J. K. (2013). Regiospecific modifications of naringenin for astragalin production in Escherichia coli. Biotechnol. Bioeng. 110, 2525-2535. doi: 10.1002/bit.24919

Pandey, R. P., Parajuli, P., Chu, L. L., Darsandhari, S., and Sohng, J. K. (2015). Biosynthesis of amino deoxy-sugar-conjugated flavonol glycosides by engineered Escherichia coli. Biochem. Eng. J. 101, 191-199. doi: 10.1016/j.bej.2015.05.017

Pandey, R. P., Parajuli, P., Koffas, M. A., and Sohng, J. K. (2016). Microbial production of natural and non-natural flavonoids: pathway engineering, directed evolution and systems/synthetic biology. Biotechnol. Adv. 34, 634-662. doi: 10.1016/j.biotechadv.2016.02.012

Pandey, R. P., Parajuli, P., Shin, J. Y., Lee, J., Lee, S., Hong, Y. S., et al. (2014). Enzymatic biosynthesis of novel resveratrol glucoside and glycoside derivatives. Appl. Environ. Microbiol. 80, 7235-7243. doi: 10.1128/aem.02076-14

Park, S.-H., Park, H.-Y., Sohng, J. K., Lee, H. C., Liou, K., Yoon, Y. J., et al. (2009). Expanding substrate specificity of GT-B fold glycosyltransferase via domain swapping and high-throughput screening. Biotechnol. Bioeng. 102, 988-994. doi: 10.1002/bit.22150

Shinde, P. B., Han, A. R., Cho, J., Lee, S. R., Ban, Y. H., Yoo, Y. J., et al. (2013). Combinatorial biosynthesis and antibacterial evaluation of glycosylated derivatives of 12-membered macrolide antibiotic YC-17. J. Biotechnol. 168, 142-148. doi: 10.1016/j.jbiotec.2013.05.014

Song, M. C., Kim, E., Ban, Y. H., Yoo, Y. J., Kim, E. J., Park, S. R., et al. (2013). Achievements and impacts of glycosylation reactions involved in natural product biosynthesis in prokaryotes. Appl. Microbiol. Biotechnol. 97, 5691-5704. doi: 10.1007/s00253-013-4978-7

Stupp, G. S., von Reuss, S. H., Izrayelit, Y., Ajredini, R., Schroeder, F. C., and Edison, A. S. (2013). Chemical detoxification of small molecules by Caenorhabditis elegans. ACS Chem. Biol. 8, 309-313. doi: 10.1021/cb300520u

Thibodeaux, C. J., Melançon, C. E. III, and Liu, H. W. (2008). Natural-product sugar biosynthesis and enzymatic glycodiversification. Angew. Chem. Int. Ed Engl. 47, 9814-9859. doi: 10.1002/anie.200801204

Tiwari, P., Sangwan, R. S., and Sangwan, N. S. (2016). Plant secondary metabolism linked glycosyltransferases: An update on expanding knowledge and scopes. Biotechnol. Adv. 34, 714-739. doi: 10.1016/j.biotechadv.2016.03.006

Weymouth-Wilson, A. C. (1997). The role of carbohydrates in biologically active natural products. Nat. Prod. Rep. 14, 99-110. doi: 10.1039/np9971400099

Williams, G. J., Yang, J., Zhang, C., and Thorson, J. S. (2011). Recombinant E. coli prototype strains for in vivo glycorandomization. ACS Chem. Biol. 6, 95-100. doi: $10.1021 / \mathrm{cb} 100267 \mathrm{k}$

Williams, G. J., Zhang, C., and Thorson, J. S. (2007). Expanding the promiscuity of a natural-product glycosyltransferase by directed evolution. Nat. Chem. Biol. 3, 657-662. doi: 10.1038/nchembio.2007.28

Yang, G., Rich, J. R., Gilbert, M., Wakarchuk, W. W., Feng, Y., and Withers, S. G. (2010). Fluorescence activated cell sorting as a general ultra-high-throughput screening method for directed evolution of glycosyltransferases. J. Am. Chem. Soc. 132, 10570-10577. doi: 10.1021/ja104167y

Yoo, S. M., Na, D., and Lee, S. Y. (2013). Design and use of synthetic regulatory small RNAs to control gene expression in Escherichia coli. Nat. Protoc. 8, 1694-1707. doi: 10.1038/nprot.2013.105

Zhang, C., Griffith, B. R., Fu, Q., Albermann, C., Fu, X., Lee, I. K., et al. (2006). Exploiting the reversibility of natural product glycosyltransferase-catalyzed reactions. Science 313, 1291-1294. doi: 10.1126/science.1130028

Zhang, J., Singh, S., Hughes, R. R., Zhou, M., Sunkara, M., Morris, A. J., et al. (2014). A simple strategy for glycosyltransferase-catalyzed aminosugar nucleotide synthesis. Chembiochem 15, 647-652. doi: 10.1002/cbic.201300779

Conflict of Interest Statement: The authors declare that the research was conducted in the absence of any commercial or financial relationships that could be construed as a potential conflict of interest.

Copyright (๑ 2016 Pandey and Sohng. This is an open-access article distributed under the terms of the Creative Commons Attribution License (CC BY). The use, distribution or reproduction in other forums is permitted, provided the original author(s) or licensor are credited and that the original publication in this journal is cited, in accordance with accepted academic practice. No use, distribution or reproduction is permitted which does not comply with these terms. 